\title{
Condition-based maintenance of wind power generation systems considering different turbine types and lead times
}

\author{
Department of Mechanical Engineering, \\ University of Alberta, \\ Edmonton, Alberta, T6G 2G8, Canada \\ E-mail: fangfang@ualberta.ca \\ E-mail: ztian@ualberta.ca \\ *Corresponding author
}

Fangfang Ding and Zhigang Tian*

\author{
Abeer Amayri \\ Department of Mechanical and Industrial Engineering, \\ Concordia University, \\ 1515 Ste-Catherine Street West, \\ Montreal, H3G 2W1, Canada \\ E-mail: abeer.amayri@gmail.com
}

\begin{abstract}
Condition monitoring measurements, such as vibration data, acoustic emission data, oil analysis data, power voltage and current data, etc., can be obtained from wind turbine components and be utilised to evaluate and predict the health conditions of the components and the turbines. The objective of condition-based maintenance (CBM) is to optimise the predictive maintenance activities based on the condition monitoring and prediction information to minimise the overall costs of wind power generation systems. In existing work, all the wind turbines are assumed to be of the same type and the lead times of different components are assumed to be constant. This is not the case in many practical applications. In this paper, we develop a CBM approach for wind turbine systems considering different types of wind turbines in a wind farm and different lead times for different turbine components, which lead to more accurate modelling of CBM activities in actual wind farms. In the proposed CBM approach, we present a new CBM policy involving two design variables for each turbine type, a method for turbine failure probability evaluation considering different lead times and a CBM cost evaluation method. Numerical examples are provided to demonstrate the proposed CBM approach.
\end{abstract}

Keywords: condition-based maintenance; CBM; wind turbine; predictive maintenance; turbine types; lead time; optimisation.

Reference to this paper should be made as follows: Ding, F., Tian, Z. and Amayri, A. (2014) 'Condition-based maintenance of wind power generation systems considering different turbine types and lead times', Int. J. Strategic Engineering Asset Management, Vol. 2, No. 1, pp.63-79. 
Biographical notes: Fangfang Ding is currently a $\mathrm{PhD}$ candidate in the Department of Mechanical Engineering, University of Alberta, Canada. She received her Master's in Quality Systems Engineering in 2011 from Concordia University. Her research is focused on wind energy systems, maintenance management and condition monitoring.

Zhigang Tian is currently an Associate Professor in the Department of Mechanical Engineering, University of Alberta, Canada. He received his $\mathrm{PhD}$ in Mechanical Engineering in 2007 at the University of Alberta, Canada; MS in 2003 and BS in 2000, both in Mechanical Engineering at Dalian University of Technology, China. His research interests focus on prognostics, condition-based maintenance, reliability, renewable energy systems, condition monitoring, signal processing, finite element modelling and optimisation. He is a member of IIE and INFORMS. He received the Best Paper Award for Quality Control and Reliability in the 2005 IIE Industrial Engineering Research Conference. He is also the recipient of the 2011 Petro-Canada Young Innovator Award (Technology, Industry and the Environment).

Abeer Amayri is currently a $\mathrm{PhD}$ student in the Department of Mechanical and Industrial Engineering at Concordia University, Canada. She obtained her Master's in Quality Systems Engineering in 2011 from Concordia University.

\section{Introduction}

Costs for operation and maintenance represent a large portion of the life cycle costs of wind power generation systems (Martinez et al., 2009; Hau, 2006) and it is critical to improve reliability and optimise maintenance management of wind turbines for the reduction of total costs (Tavner et al., 2007; Krokoszinski, 2003; Sen, 1997). For example, the Canadian wind energy industry has been growing very rapidly and it is expected that wind energy will satisfy $20 \%$ of Canada's electricity demand by 2025 . Operation and maintenance costs account for $25-30 \%$ of the wind energy generation cost. Currently, most wind farms use reactive maintenance, also known as corrective maintenance and time-based preventive maintenance strategies (Jardine and Tsang, 2006). In reactive maintenance, maintenance actions are performed only after failure occurs. In time-based preventive maintenance, preventive maintenance actions are performed typically at constant-intervals (CI); say every six months and the failed components are maintained once they fail. To ensure system availability and reduce the cost due to loss of productivity, the preventive maintenance interval has to be small enough, which leads to the increase of the total maintenance costs.

Currently, the wind energy industry is switching from reactive maintenance and time-based preventive maintenance strategies to condition-based maintenance (CBM) strategy by more effectively utilising condition monitoring information. In CBM, condition monitoring measurements, such as vibration data, acoustic emission data, oil analysis data, power voltage and current data, can be obtained from wind turbine components and be utilised to evaluate and predict the health conditions of the components and the turbines (Liu et al., 2010; Hameed et al., 2010; Caselitz and Giebhardt, 2005; Banjevic et al., 2001; Gebraeel et al., 2005; Tian, 2009). The objective of CBM is to optimise the predictive maintenance activities based on the condition monitoring and prediction information to minimise the overall costs of wind power 
generation systems. Newly deployed turbines are typically equipped with condition monitoring sensors. For legacy turbines, operators and manufacturers are also trying to install such sensors on many of these turbines. There is a significant and growing need to develop effective methods for optimising maintenance activities by fully utilising the available condition monitoring information. Nilsson and Bertling (2007) proposed a life cycle cost approach for evaluating the financial benefits using condition monitoring system, a tool required in CBM. Byon and Ding (2010) applied a multi-state Markov decision mechanism in estimating the wind turbine degradation process, based on which the optimal maintenance scheme is determined. They also investigated the simulation of wind farm operations and maintenance using discrete event simulation (DEVS) (Byon et al., 2011). Sorensen (2009) investigated the framework for risk-based planning of operation and maintenance for offshore wind turbines, which are typically difficult to access.

There are typically multiple wind turbines in a wind farm, which is typically located at a remote site. A wind farm can be considered to be a system consisting of multiple wind turbines and there are multiple components including main bearing, gearbox, generator, shafts, etc., in each wind turbine. Thus, there are economic dependencies among wind turbines and their components in a wind farm. More specifically, there are fixed costs for sending a maintenance team to the wind farm and for stopping a turbine for maintenance. Thus, it may be cheaper to take the opportunities to maintain multiple turbines and multiple components in a turbine at the same time, based on the evidence presented in the condition monitoring data. Tian et al. (2011) developed a CBM approach for wind energy systems considering such economic dependencies. In their work, they focused on a homogeneous wind turbine fleet made by the same manufacturer and assuming constant lead time for all turbine components. However, it is very likely a wind farm owner acquires different capacities of wind turbines from the same manufacturer or different brands from different manufacturers. Different types of wind turbines have different types of capacities and typically different types of turbine components. Thus, the CBM decision making criteria are expected to be different for different types of turbines. In addition, different turbine components are likely from different suppliers and thus have different lead times.

In this paper, we develop a CBM approach for wind power generation systems considering different types of wind turbines in a wind farm and different lead times for different turbine components which lead to more accurate modelling of CBM activities in actual wind farms. In the proposed CBM approach, we present a new CBM policy involving two design variables for each turbine type, a method for turbine failure probability evaluation considering different lead times and a CBM cost evaluation method. Numerical examples will be provided to demonstrate the proposed CBM approach.

\section{The proposed CBM approach}

The proposed CBM approach for wind power generation systems is presented in this section. The proposed CBM policy considering different types of turbines and different lead times is described first, followed by the summary of the health condition prediction method used and the method for calculating the condition failure probability. The cost 
evaluation method for the CBM policy, which is a key part of the approach is presented in the last subsection.

\subsection{The proposed CBM policy}

Suppose wind turbine components are continuously monitored in the wind farm. At a certain inspection point, condition monitoring data can be collected and analysed for health condition prediction and thus for the computation of component and turbine level condition failure probability values during the lead time, which will be discussed in details later. Maintenance decisions are made based on the CBM policy and the condition monitoring data. Suppose there are totally $K$ types of turbines in the wind farm. The proposed CBM policy for wind power generation systems considering different types of turbines and different lead times is given as follows.

- Perform failure replacement if a component fails.

- Send a maintenance team to the wind farm if any wind turbine in the wind farm is determined to be maintained.

- Perform preventive replacements on components in wind turbine $W T_{k, n}$ if $P r_{k, n}>d_{1}^{k}$, where $P r_{k, n}$ is the conditional failure probability of the wind turbine, which belongs to type $k$, and $d_{1}^{k}$ is the pre-specified level 1 failure probability threshold value at the turbine level for turbine type $k$.

- If turbine $W T_{k, n}$ is to be performed preventive replacement on, perform preventive replacement on its components in order to bring the turbine failure probability down to below $d_{2}^{k}$, which is called the level 2 failure probability threshold value at the turbine for turbine type $k$.

- If a turbine has a failed component, perform all the possible failure and preventive replacements for the turbine at the same time.

It can be seen that the CBM policy is determined by the two failure probability threshold values, $d_{1}^{k}$ and $d_{2}^{k}$, for each turbine type $k$ and thus there are $2 K$ failure probability threshold values in total.

\subsection{Health condition prediction for wind turbine components}

Health condition prediction aims to predict wind turbine component future health conditions as well as the remaining useful lives. At a certain inspection point, the condition monitoring measurements are collected and the health condition prediction methods can be used to obtain the predicted failure time value and the associated uncertainty, in another word, the predicted failure time distribution. Various prognostics methods can be used for wind turbine components, including various model-based methods and data-driven methods (Tian et al., 2010). The proposed CBM approach for wind power generation systems can work with any type of prognostics methods, as long as the predicted failure time distribution can be obtained at each certain inspection point 
based on the condition monitoring data. In this work, it is assumed that at a certain inspection point, the predict uncertainty (error) follows normal distribution; hence the predicted failure time for a wind turbine component also follows normal distribution (Tian et al., 2011). The predicted failure time is generally much larger than the standard deviation and thus the probability that the failure time take negative value due to the use of normal distribution can be safely ignored. For component $m$ in turbine $n$ of type $k$, the mean of the predicted failure time is denoted by $T P_{k, n, m}$ and the corresponding standard deviation is denoted by $\sigma_{k, n, m}=\sigma_{p} \cdot T P_{k, n, m}$, where $\sigma_{p}$ is the standard deviation of prediction error. Thus at a certain inspection point, the distribution of the predicted failure time, denoted by $T_{p}$, can be represented by:

$$
T_{p} \sim N\left(T P_{k, n, m}, \sigma_{p} \cdot T P_{k, n, m}\right) .
$$

\subsection{Evaluation of turbine conditional failure probability}

As mentioned in Section 2.1 when the CBM policy is introduced, the CBM decision making requires the conditional failure probability values at the turbine component level and the turbine level. For component $m$ in turbine $n$ of type $k$, the conditional failure probability during its lead time, denoted by $\operatorname{Pr}_{k, n, m}$, can be formulated as:

$$
P r_{k, n, m}=\frac{\int t_{t_{k, n, m}}+L_{k, m} \frac{1}{\sigma_{k, n, m} T P_{k, n, m} \sqrt{2 \pi}} e^{-\frac{1}{2}\left(\frac{x-T P_{k, n, m}}{\sigma_{k, n, m} T P k, n, m}\right)^{2}} d x}{\int_{\substack{t_{k, n, m} \\ \sigma_{k, n, m} T P_{k, n, m} \sqrt{2 \pi}}} e^{-\frac{1}{2}\left(\frac{x-T P_{k, n, m}}{\sigma_{k, n, m} T P k, n, m}\right)^{2}} d x}
$$

where $t_{k, n, m}$ is the age of the component at the current inspection point. $L_{k, m}$ is the lead time for component $m$ in type $k$ turbine, which is defined as the interval between the time maintenance decision is made and the time when the maintenance is completed for the component. Basically, $\operatorname{Pr}_{k, n, m}$ is the conditional probability that the component fails during the lead time given that it is still working at the current inspection point, based on the predicted failure time distributions.

At the turbine level, the turbine is considered to be a series system. That is, a wind turbine is considered failed if any of its components is failed. At a certain inspection point, the conditional failure probability of a wind turbine can be determined as follows:

$$
\operatorname{Pr}_{k, n}=1-\prod_{m=1}^{M_{k}}\left(1-\operatorname{Pr}_{k, n, m}\right)
$$

where $M_{k}$ is the total number of components considered for the turbine of type $k$.

\subsection{Cost evaluation for the CBM policy}

According to the previously described CBM policy, the optimal failure probability thresholds of each type $k$ can be determined so that the corresponding total expected maintenance cost per unit time per turbine, denoted by $C_{E}$ can be minimised. The objective function for the CBM optimisation problem can be given as: 


$$
\begin{aligned}
& \min C_{E}\left(d_{1}^{1}, d_{2}^{1}, d_{1}^{2}, d_{2}^{2}, \ldots, d_{1}^{K}, d_{2}^{K}\right) \\
& \text { s.t. } \\
& \quad 0<d_{2}^{k}<d_{1}^{k}<1
\end{aligned}
$$

where $d_{1}^{1}$ and $d_{2}^{1}, d_{1}^{2}$ and $d_{2}^{2}, \ldots, d_{1}^{K}$ and $d_{2}^{K}$ represent two level failure thresholds in type $1,2, \ldots, K$, respectively.

The key step in solving this optimisation problem is to calculate the cost $C_{E}$ given a certain CBM policy, defined by the failure probability threshold values $d_{1}^{1}, d_{2}^{1}, d_{1}^{2}, d_{2}^{2}, \ldots$, and $d_{1}^{K}, d_{2}^{K}$ for each type $k$. Due to the significant complexity in the cost evaluation; it is very hard to develop an analytical method or accurate numerical method for the cost evaluation of the CBM policy. Thus, in this paper, a simulation method is developed to evaluate the cost due to its flexibility and capability of solving complex problems. The procedure of the cost evaluation is outlined in Figure 1. The detailed explanations are given in the rest of this section.

Figure 1 Cost evaluation procedure for the proposed CBM policy

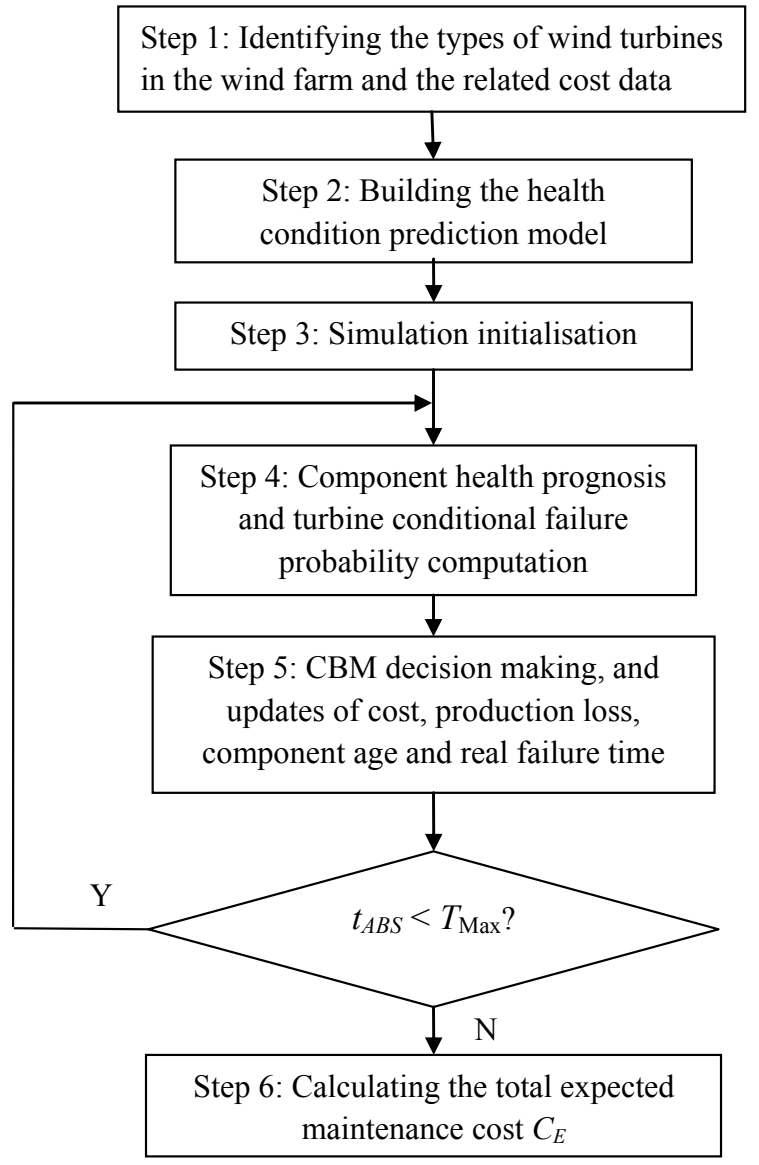




\subsubsection{Step 1}

Suppose there are $K$ types of wind turbines in the wind farm, which are categorised by rated power and model. In each type, $N_{k}$ wind turbines and $M_{k}$ critical components are considered for each turbine. For the component $m$ in each type of wind turbines, specify the maintenance lead time $L_{k, m}$, cost values including the failure replacement $\operatorname{cost} c_{k, f, m}$ and the variable preventive replacement $\operatorname{cost} c_{k, p, m}$. In addition, specify the fixed cost of maintaining a certain wind turbine $c_{p, T}$ and the fixed cost of sending a maintenance team to the wind farm $c_{\text {farm }}$.

\subsubsection{Step 2}

Prognostics methods are used to predict component failure time distributions before implementing CBM. The Weibull distribution is used for describing the turbine component lifetime distribution for the population. Based on the available historical failure and suspension data, the distribution scale parameter $\left(\alpha_{k, m}\right)$ and shape parameter $\left(\beta_{k, m}\right)$ can be estimated for each component $m$ in type $k$ turbines. For each type of turbine, based on historical degradation data, the health prediction model can be built.

\subsubsection{Step 3}

Simulation initialisation. In this step, the maximum simulation iteration $\left(T_{\mathrm{Max}}\right)$ and the inspection interval $T_{I}$ are specified. The accuracy of the results is affected by the number of iterations. $T_{I}$ is set to be a small value, e.g., ten days, so that continuous monitoring can be approximately achieved. The total $\operatorname{cost} C_{T}$ and time set $t_{A B S}$ are set to be zero before starting the simulation. Generate the real failure time $T L_{k, n, m}$ of component $m$ in turbine $n$ of type $k$ by sampling Weibull distribution with parameter $\left(\alpha_{k, m}\right)$ and $\left(\beta_{k, m}\right)$. At time $t_{A B S}=0$ for all components, set the age $t_{k, n, m}=0$. The total cost and the age of component will be updated during the simulation process.

\subsubsection{Step 4}

Component health prognosis and turbine failure probability calculation. At a certain inspection point when $t_{A B S}>0$ the current age and real failure time of component $m$ are known, which are $t_{k, n, m}$ and $T L_{k, n, m}$. The predicted failure time distribution is the output of component health prognosis and it can be described using normal distribution $N\left(T P_{k, n, m}, \sigma_{k, n, m}^{2}\right)$, as discussed earlier in Section 2.2. Thus, the conditional failure probability during the lead time $L_{k, m}$ for the component $m$ in the turbine $n$ of type $k$ can be calculated using equation (2) and the failure probability for each turbine can be calculated using equation (3) with its components' conditional failure probabilities.

\subsubsection{Step 5}

CBM decision making and updates of cost, production loss, component age and real failure time. At each inspection point $t_{A B S}$ the CBM decisions can be made based on different specific situations, which are discussed in details as follows.

1 If $t_{k, n, m}>T L_{k, n, m}$, it indicates that a component has failed and a failure replacement will be performed on the component. The loss of productivity before the inspection point occurs, which can be generally calculated as follows: 


$$
C_{\text {Loss }}=\text { Power Rate } \times 24 \text { hrs } \times \frac{\$ 50}{M W} \times 30 \% \times \text { Stop Days }
$$

For the wind turbine with failed components, the number of stop days before the inspection point is given as:

$$
T_{\text {LossBefore }, k, n}=\operatorname{Max}\left\{\left(t_{k, n, m}-T L_{k, n, m}\right), 0\right\}, \text { for all } m
$$

Using above two equations, we can obtain $C_{\text {LossBefore }, k, n}$ for the failed turbine $n$ of type $k$. The cost increase due to failure replacements can be formulated as:

$$
\Delta C_{T, F}=\sum_{k=1}^{K} \sum_{n=1}^{N_{k}} \sum_{m=1}^{M_{k}} I F_{k, n, m} c_{k, f, m}
$$

where $I F_{k, n, m}=1$ if the component $m$ fails, otherwise it equals 0 . The cost due to loss of productivity will be added later.

2 For wind turbine $n$, if $P r_{k, n}>d_{1}^{k}$, perform preventive replacement on its components with higher conditional failure probabilities until the turbine failure probability is lower than $d_{2}^{k}$, the lower level failure probability threshold. Accordingly, the preventive replacement costs are incurred. The cost increase due to preventive replacements can be formulated as:

$$
\Delta C_{T, P}=\sum_{k=1}^{K} \sum_{n=1}^{N_{k}}\left(\sum_{m=1}^{M_{k}} I P_{k, n, m} c_{k, p, m}+I T_{n} c_{k, p, T}\right)
$$

where $I P_{k, n, m}=1$ if a preventive replacement is to be performed on the component $m$, otherwise equals $0 ; I T_{n}=1$ if preventive replacements are performed but no failure replacements are performed in the turbine $n$.

3 For a wind turbine with failed components, it is assumed that the turbine won't start to be used until all the failed components have been replaced and all the required preventive replacements have been performed as well. Thus, the number of the stop days after the current inspection point is equal to the maximum of the lead times of the components that are to be replaced.

$$
T_{\text {LossAfter }, k, n}=\operatorname{Max}\left\{L_{k, m}\right\} \text {, for all components } m s \text { that are to be replaced }
$$

where $L_{k, m}$ denotes the lead times of all the components to be maintained in the turbine $n$ of type $k$, including preventive maintenance and failure maintenance. Thus, we can calculate the cost due to the loss of productivity as follows:

$$
\Delta C_{T, \text { Loss }}=C_{\text {Loss }}=\text { Power Rate } \times 24 \mathrm{hrs} \times \frac{\$ 50}{M W} \times 30 \% \times\left(T_{\text {Loss }, k, n}\right)
$$

where $T_{\text {Loss }, k, n}=T_{\text {LossAfter }, k, n}+T_{\text {LossBefore }, k, n}$, representing the total number of stop days for the turbine.

4 In addition, there is the fixed cost of sending a maintenance team to the wind farm:

$$
\Delta C_{T, \text { Farm }}=I F_{\text {farm }} C_{\text {farm }}
$$

where $I F_{\text {farm }}=1$ if preventive replacements or failure replacements are to be performed in the wind farm, otherwise equals 0 . 
5 At the current inspection point $t_{A B S}$ if any replacement is to be performed, the next inspection interval will be updated as:

$$
L_{\text {farm }}=\operatorname{Max}\left\{L_{k, n, m}\right\}
$$

where $L_{k, n, m}$ denotes all the lead time values of the components to be determined for maintenance in the wind farm. The next inspection time will move to the point when $L_{f a r m}$ has passed, i.e.,

$$
t_{A B S}=t_{A B S}+L_{f a r m}
$$

Otherwise, we will move the next inspection point with regular inspection interval, i.e.,

$$
t_{A B S}=t_{A B S}+T_{I}
$$

At the new inspection time, if a failure or preventive replacement is decided to be performed on component $m$ in turbine $n$ of type $k$, regenerate a new real failure time by sampling Weibull distribution with parameters $\alpha_{k, m}$ and $\beta_{k, m}$.

If $t_{A B S}<T_{\text {Max }}$, repeat Step 3, 4 and 5.

\subsubsection{Step 6}

The total expected maintenance cost calculation. When it goes to the maximum simulation iteration $T_{\mathrm{Max}}$, i.e., when $t_{A B S}=T_{\mathrm{Max}}$, the simulation procedure is completed. The total expected maintenance cost per unit time per turbine for the wind farm is therefore:

$$
C_{E}=\frac{C_{T}}{T_{\mathrm{Max}} \cdot \sum_{k=1}^{K} N_{k}}
$$

\section{Examples}

In this section, we use some examples to demonstrate the proposed CBM approach for a wind farm, where two types of turbines are installed, with three turbines belong to type 1, low capacity and the other three turbines belong to type 2, high capacity. To simplify the discussion, we study four key components in each wind turbine: rotor, main bearing, gearbox and generator. The structure of wind turbines is shown in Figure 2 (National Instruments Products for Wind Turbine Condition Monitoring, 2010). We also compare the results using the proposed approach with the widely used CI preventive maintenance method and the method considering an approximate constant lead time for all the turbine components. 
Figure 2 Key wind turbine components considered in the example (see online version for colours)

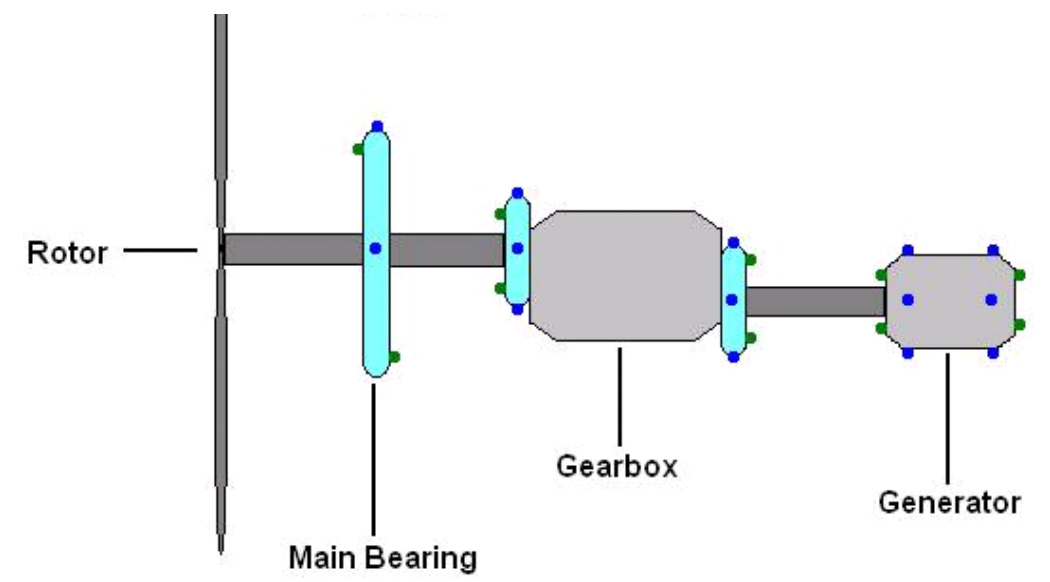

Source: National Instruments Products for Wind Turbine Condition Monitoring (2010)

\subsection{The proposed CBM approach}

\subsubsection{Example introduction}

In the example, two types of turbine are installed in the wind farm, which are low capacity turbines at $2 \mathrm{MW}$ and high capacity turbines at $5 \mathrm{MW}$ respectively. Thus, the costs and failure distribution parameter values, as well as the lead times of components, vary with different type of wind turbine. Assume the Weibull distributions are appropriate to present the component failures of the populations and the Weibull parameters and maintenance lead times corresponding to each type of turbines are given in Table 1. The cost data are given in Table 2, including the failure replacement costs for the components, the fixed and variable preventive replacement costs with respect to each type and the fixed cost of sending a maintenance team to the wind farm. All data used in this example are specified based on the data given in our previous study in Tian et al. (2011),

which are constructed based on reported data in the literature (WindStats Newsletter, 2008-2009; Guo et al., 2009; Fingersh et al., 2006). In addition, the inspection interval is set at ten days.

Table 1 Weibull failure time distribution parameters and maintenance lead times for major components

\begin{tabular}{|c|c|c|c|c|c|}
\hline \multirow{2}{*}{ Component } & \multicolumn{2}{|c|}{ Scale parameter $\alpha$ (days) } & \multirow{2}{*}{ Shape parameter $\beta$} & \multicolumn{2}{|c|}{ Lead time } \\
\hline & Type 1 & Type 2 & & Type 1 & Type 2 \\
\hline Rotor & 3,000 & 6,000 & 3.0 & 30 & 45 \\
\hline Main bearing & 3,750 & 7,500 & 2.0 & 10 & 15 \\
\hline Gearbox & 2,400 & 4,800 & 3.0 & 25 & 30 \\
\hline Generator & 3,300 & 6,600 & 2.0 & 15 & 20 \\
\hline
\end{tabular}


Table 2 Failure replacement and preventive maintenance costs for major components

\begin{tabular}{|c|c|c|c|c|c|c|c|c|}
\hline \multirow[t]{2}{*}{ Component } & \multicolumn{2}{|c|}{$\begin{array}{c}\text { Failure } \\
\text { replacement } \\
\text { cost }(\$ 1,000)\end{array}$} & \multicolumn{2}{|c|}{$\begin{array}{c}\text { Variable preventive } \\
\text { maintenance cost } \\
(\$ 1,000)\end{array}$} & \multicolumn{2}{|c|}{$\begin{array}{l}\text { Fixed preventive } \\
\text { maintenance cost } \\
\qquad(\$ 1,000)\end{array}$} & \multicolumn{2}{|c|}{$\begin{array}{l}\text { Fixed cost to the } \\
\text { wind farm } \\
(\$ 1,000)\end{array}$} \\
\hline & Type 1 & Type 2 & Type 1 & Type 2 & Type 1 & Type 2 & Type 1 & Type 2 \\
\hline Rotor & 112 & 224 & 28 & 56 & \multirow{4}{*}{25} & \multirow{4}{*}{50} & \multirow{4}{*}{50} & \\
\hline Main bearing & 60 & 120 & 15 & 30 & & & & \\
\hline Gearbox & 152 & 304 & 38 & 76 & & & & \\
\hline Generator & 100 & 200 & 25 & 50 & & & & \\
\hline
\end{tabular}

\subsubsection{The health condition prediction method}

In this example, the artificial neural network (ANN)-based prognostics method developed by Tian et al. (2010) is used. The structure of the ANN model used in this approach is shown in Figure 3. The inputs of the ANN are the component age values, denoted by $t_{i}$ and $t_{i-l}$, for the current inspection point $i$ and the previous inspection point and the condition monitoring measurements at these inspection points, denoted by $z_{i}^{1}$ and $z_{i-1}^{1}$ for measurement 1 and $z_{i}^{2}$ and $z_{i-1}^{2}$ for measurement 2. Different types of measurements might be used depending on the problems. The output of the ANN model, denoted by $P_{i}$, is the life percentage at current inspection time (Tian et al., 2010, 2011). Once trained based on historical data, the ANN prediction model can be used to predict the remaining life based on the component age and the condition monitoring data. For example, at a certain inspection point, if the current age of the component is 400 days and the predicted life percentage obtained using the ANN prediction model is $80 \%$, the predicted failure time would be $400 / 80 \%=500$ days. The uncertainty associated with the predicted failure time can be estimated based on the prediction errors obtained during the ANN training and testing processes. Thus, the predicted failure time distribution for components can be obtained, as the results can be described in equation (1).

Figure 3 Structure of the ANN health condition prediction model

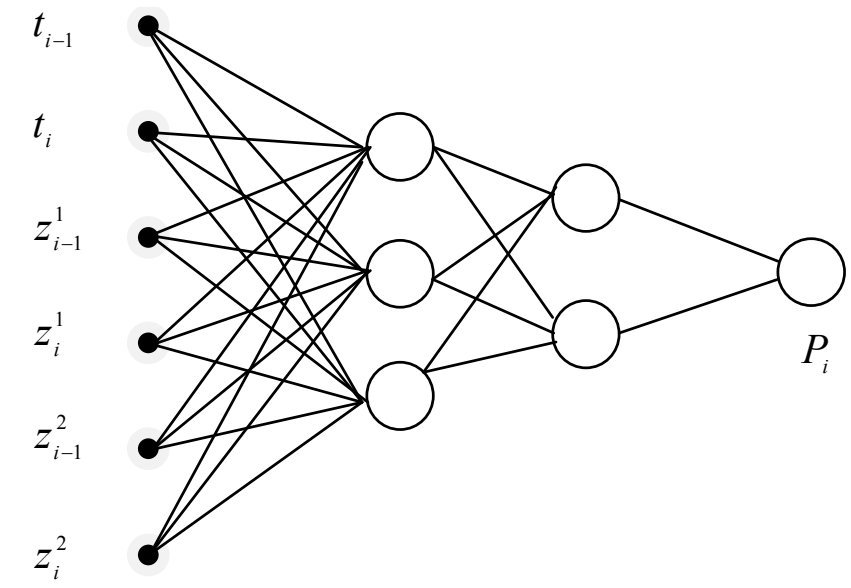

Source: Stevens (2006) 
For this example, the above-mentioned ANN-based prediction method is applied to predict the failure time distributions of the components. The standard deviations of the ANN life prediction errors are required to establish the associated prediction uncertainties (Tian et al., 2010; Stevens, 2006) and the values of the standard deviations of each type system are given in Table 3.

Table 3 ANN life percentage prediction error standard deviation values for major components

\begin{tabular}{lcc}
\hline \multirow{2}{*}{ Component } & \multicolumn{2}{c}{ Standard deviation } \\
\cline { 2 - 3 } & Type 1 & Type 2 \\
\hline Rotor & 0.12 & 0.15 \\
Main bearing & 0.10 & 0.13 \\
Gearbox & 0.10 & 0.13 \\
Generator & 0.12 & 0.15 \\
\hline
\end{tabular}

\subsubsection{The CBM optimisation results}

The total maintenance cost can be evaluated using the proposed simulation method described in Section 2.4. After all the costs have been calculated, we search and find out the minimum cost and the corresponding variable values, these are therefore the optimal results. There are four design variables in this optimisation and thus it is hard to visualise the cost versus the conditional probability threshold values. It is found that the total maintenance cost is affected by the four failure probability threshold values for the two types of wind turbines and the optimal CBM policy can be found corresponding to the lowest cost. After performing optimisation, the optimal CBM policy can be obtained and the optimal threshold failure probability values for Type-1 turbines are: $d_{1}^{1}=0.063$, $d_{2}^{1}=1.36 \times 10^{-4}$ and the optimal threshold failure probability values for Type- 2 turbines are: $d_{1}^{2}=0.063, d_{2}^{2}=6.3 \times 10^{-6}$. The optimal expected maintenance cost per unit of time per turbine is $84.8 \$$ day/turbine and thus for the whole farm with six turbines, the optimal maintenance cost per unit of time is $508.8 \$$ day.

\subsubsection{Comparison with the CBM approach considering constant lead time}

The CBM approach developed in Tian et al. (2011) assumes constant lead time for all the turbine components. When the constant lead time is determined, the maximum lead time among all the components is used and thus the maintenance work can be considered completed at the end of the lead time for any component. For a wind farm with different types of turbines and different components, the constant lead time assumption is of course approximates. The CBM approach developed in this work provides an accurate representation of the wind farm and the optimal maintenance cost is $84.8 \$$ day/turbine, as described in Section 3.1.3. If we apply the constant lead time assumption to this example, the lead time would be 45 days, which is the maximum of all the lead time values, according to Table 1. At the optimal solution obtained in Section 3.1.3, where $d_{1}^{1}=0.063, d_{2}^{1}=1.36 \times 10^{-4}$ and $d_{1}^{2}=0.063, d_{2}^{2}=6.3 \times 10^{-6}$, the cost value obtained under the constant lead time assumption is $90.02 \$ /$ day/turbine. This indicates that there is approximately $6.2 \%$ deviation from the real cost value if we use the constant lead time assumption. 


\subsection{Comparative study with the CI policy}

In this section, we compare the proposed CBM approach with the CI policy, which is a type of time-based preventive maintenance policy and is currently widely used in the wind energy industry. The main objective of the CI method is to determine the optimal preventive replacement interval, denoted by $t_{C I}$, to minimise the total expected cost per unit time. In this section, we investigate the CI maintenance policy using the same lifetime distributions for the components, as given in Table 1. The total expected maintenance cost per unit time for the wind farm can be obtained based on the method in Jardine and Tsang (2006) and the equation is given as:

$$
C\left(t_{C I}\right)=\frac{\sum_{k=1}^{K} N_{k} \cdot \sum_{m=1}^{M_{k}}\left(C_{p, k, n, m}^{C I}+C_{f, k, n, m}^{C I} H_{m, k}\left(t_{C I}\right)\right)}{t_{C I}}
$$

where $C\left(t_{C I}\right)$ denotes the total preventive maintenance cost, $C_{f, k, n, m}^{C I}$ is the total cost of a failure replacement for component $m$ in turbine $n$ of type $k$ and $C_{p, k, n, m}^{C I}$ is the total cost of a preventive replacement for component $m$ in turbine $n$ of type $k . H_{m, k}\left(t_{C I}\right)$ is the expected number of failures for component $m$ in type $k$ turbine in interval $\left(0, t_{C I}\right)$, which can be evaluated using the recursive procedure outline in Jardine and Tsang (2006). Thus, the total expected maintenance cost per unit time per turbine, denoted by $C_{E}\left(t_{C I}\right)$, can be obtained as:

$$
C_{E}\left(t_{C I}\right)=\frac{C\left(t_{C I}\right)}{\sum_{k=1}^{K} N_{k}}
$$

The optimal interval $t_{C I}$ can be obtained to minimise the expected maintenance cost per unit time using the optimisation tool.

To ensure a fair comparison, the cost data is constructed based on those in Section 3.1. Since the fixed cost of sending the maintenance team to the wind farm, $c_{\text {Farm }}$ ' is incurred at each time of failure occurrence; it is regarded as a part of the failure replacement cost. The loss of productivity cost is also considered through the lead time. Thus, the failure replacement cost for component $m$ in turbine $n$ of type $k$ can be given as:

$$
C_{f, k, n, m}^{C I}=c_{k, f, m}+C_{f a r m}+\text { Power Rate } \times 24 \mathrm{hrs} \times \frac{\$ 50}{M W} \times 30 \% \times L_{k, m}
$$

At each time of preventive replacement, in addition to sharing the fixed cost on the farm level $c_{\text {farm }}$, the individual component's preventive maintenance cost also shares the turbine level fixed preventive maintenance cost in Table 2. In addition to that, the loss of productivity cost is incurred and is distributed among all the components in the turbine, since the components are replaced simultaneously. Therefore, the preventive replacement cost for component $m$ in turbine $n$ of type $k$ can be calculated as: 


$$
\begin{aligned}
C_{p, k, n, m}^{C I}=c_{p, k, m} & +\frac{C_{p, T}+\text { Power Rate } \times 24 \mathrm{hrs} \times \frac{\$ 50}{M W} \times 30 \% \times \operatorname{Max}\left\{L_{k, m}\right\}}{M_{k}} \\
& +\frac{C_{\text {farm }}}{\sum_{k=1}^{K} n_{k} M_{k}}
\end{aligned}
$$

The calculated failure replacement and preventive maintenance costs data using equations (18) and (19) are show in Table 4.

\begin{tabular}{|c|c|c|c|c|}
\hline \multirow[t]{2}{*}{ Component } & \multicolumn{2}{|c|}{$\begin{array}{l}\text { Failure replacement cost } \\
\qquad(\$ 1,000)\end{array}$} & \multicolumn{2}{|c|}{$\begin{array}{c}\text { Preventive replacement cost } \\
\qquad(\$ 1,000)\end{array}$} \\
\hline & Type 1 & Type 2 & Type 1 & Type 2 \\
\hline Rotor & 378 & 1,084 & 90.33 & 273.08 \\
\hline Main bearing & 182 & 440 & 77.33 & 247.08 \\
\hline Gearbox & 382 & 894 & 100.33 & 293.08 \\
\hline Generator & 258 & 610 & 87.33 & 267.08 \\
\hline
\end{tabular}

Table 4 Cost data for the CI maintenance policy

The total expected maintenance cost per unit of time can be calculated using equation (17). The cost versus preventive maintenance interval is plotted and shown in Figure 4. By performing optimisation, the optimal replacement interval is found to be 3,340 days and the corresponding optimal cost is 471.89 \$day/turbine. Comparing with the optimal cost $84.8 \$ /$ day/turbine obtained using the proposed CBM approach, it can be seen that about $82 \%$ cost savings can be achieved using the proposed CBM approach.

Figure 4 Cost versus preventive maintenance interval for the CI policy (see online version for colours)

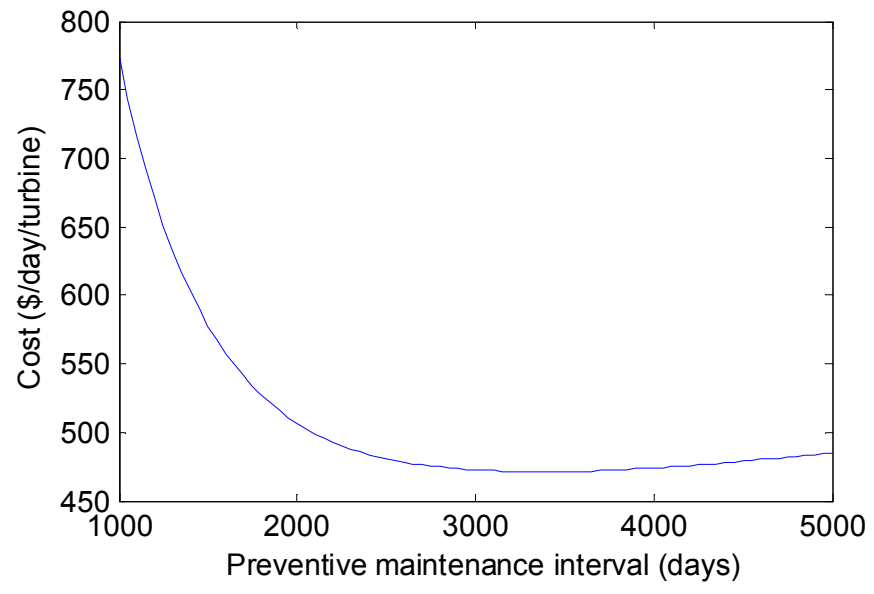




\section{Conclusions}

Currently, the wind energy industry is switching from reactive maintenance and time-based preventive maintenance strategies to CBM strategy by utilising condition monitoring information more effectively. In existing work, all the wind turbines are assumed to be of the same type and the lead times of different components are assumed to be constant. This is not the case in many practical applications. In this paper, we develop a CBM approach for wind turbine systems considering different types of wind turbines in a wind farm and different lead times for different turbine components which lead to more accurate modelling of CBM activities in actual wind farms. In the proposed CBM approach, we present a new CBM policy involving two design variables for each turbine type, a method for turbine failure probability evaluation considering different lead times and a CBM policy cost evaluation method. The numerical examples demonstrate the effectiveness of the proposed CBM approach.

\section{Acknowledgements}

This research is supported by the Natural Sciences and Engineering Research Council of Canada (NSERC) and Le Fonds québécois de la recherche sur la nature et les technologies (FQRNT).

\section{References}

Banjevic, D., Jardine, A.K.S., Makis, V. and Ennis, M. (2001) 'A control-limit policy and software for condition based maintenance optimization', INFOR, Vol. 39, No. 1, pp.32-50.

Byon, E. and Ding, Y. (2010) 'Season-dependent condition based maintenance for a wind turbine using a partially observed Markov decision process', IEEE Transactions on Power Systems, Vol. 25, No. 4, pp.1823-1834, doi: 10.1109/TPWRS.2010.2043269.

Byon, E., Pérez, E., Ding, Y. and Ntaimo, L. (2011) 'Simulation of wind farm operations and maintenance using DEVS', December, Simulation, Vol. 87, No. 12, pp.1093-1117.

Caselitz, P. and Giebhardt, J. (2005) 'Rotor condition monitoring for improved operational safety of offshore wind energy converters', ASME Trans. Journal of Solar Energy Engineering, Vol. 127, No. 2, pp.253-261, doi: 10.1115/1.1850485.

Fingersh, L., Hand, M. and Laxson, A. (2006) Wind Turbine Design Cost and Scaling Model, National Renewable Energy Laboratory technical report.

Gebraeel, N., Lawley, M., Li, R. and Ryan, J.K. (2005) 'Life distributions from component degradation signals: a Bayesian approach', IIE Transactions on Quality and Reliability Engineering, Vol. 37, No. 6, pp.543-557, doi: 10.1080/07408170590929018.

Guo, H.T., Watson, S. and Tavner, P. (2009) 'Reliability analysis for wind turbines with incomplete failure data collected from after the date of initial installation', Reliability Engineering and System Safety, Vol. 94, No. 6, pp.1057-1063, doi: 10.1016/j.ress.2008.12.004.

Hameed, Z., Ahn, S.H. and Cho, Y.M. (2010) 'Practical aspects of a condition monitoring system for a wind turbine with emphasis on its design, system architecture, testing and installation', Renewable Energy, Vol. 35, No. 5, pp.879-894, doi: 10.1016/j.renene.2009.10.031.

Hau, E. (2006) Wind Turbines: Fundamentals, Technologies, Application, Economics, Springer, Germany. 
Jardine, A.K.S. and Tsang, A.H. (2006) Maintenance, Replacement and Reliability Theory and Applications, CRC Press, Taylor \& Francis Group, USA.

Krokoszinski, H.J. (2003) 'Efficiency and effectiveness of wind farms - keys to cost optimized operation and maintenance', Renewable Energy, Vol. 28, No. 14, pp.2165-2178.

Liu, W.Y., Tang, B.P. and Jiang, Y.H. (2010) 'Status and problems of wind turbine structural health monitoring techniques in China', Renewable Energy, Vol. 35, No. 7, pp.1414-1418, doi: 10.1016/j.renene.2010.01.006.

Martinez, E., Sanz, F. and Pellegrini, S. (2009) 'Life cycle assessment of a multi-megawatt wind turbine', Renewable Energy, Vol. 34, No. 3, pp.667-673, doi: 10.1016/j.renene.2008.05.020.

National Instruments Products for Wind Turbine Condition Monitoring (2010) [online] http://www.zone.ni.com/devzone/cda/tut/p/id/7676 (accessed December 2011).

Nilsson, J. and Bertling, L. (2007) 'Maintenance management of wind power systems using condition monitoring systems - life cycle cost analysis for two case studies', IEEE Transactions on Energy Conversion, Vol. 22, No. 1, pp.223-229, doi: 10.1109/TEC.2006.889623.

Sen, Z. (1997) 'Statistical investigation of wind energy reliability and its application', Renewable Energy, Vol. 10, No. 1, pp.71-79, doi: 10.1016/0960-1481(96)00021-3.

Sorensen, J.D. (2009) 'Framework for risk-based planning of operation and maintenance for offshore wind turbines', Wind Energy, Vol. 12, No. 5, pp.493-506.

Stevens, B. (2006) EXAKT Reduces Failures at Canadian Kraft Mill [online] http://www.modec.com (accessed December 2011).

Tavner, P.J., Xiang, J. and Spinato, F. (2007) 'Reliability analysis for wind turbines', Wind Energy, Vol. 10, No. 1, pp.1-18.

Tian, Z. (2009) 'An artificial neural network method for remaining useful life prediction of equipment subject to condition monitoring', Journal of Intelligent Manufacturing, Vol. 23, No. 2, pp.227-237.

Tian, Z., Jin, T., Wu, B. and Ding, F. (2011) 'Condition based maintenance optimization for wind power generation systems under continuous monitoring', Renewable Energy, Vol. 36, No. 5, pp.1502-1509.

Tian, Z., Wong, L. and Safaei, N. (2010) 'A neural network approach for remaining useful life prediction utilizing both failure and suspension histories', Mechanical Systems and Signal Processing, Vol. 24, No. 5, pp.1542-1555, doi: 10.1016/j.ymssp.2009.11.005.

WindStats Newsletter (2008-2009) Vol. 21, No. 4-Vol. 22, No. 3, Denmark.

\section{Abbreviations}

\begin{tabular}{ll}
\hline CBM & Condition-based maintenance \\
ANN & Artificial neural network \\
CI & Constant-interval \\
\hline
\end{tabular}




\section{Nomenclature}

\begin{tabular}{|c|c|}
\hline$K$ & The number of types of wind turbines in a wind farm \\
\hline$N_{k}$ & The number of wind turbines of type $k$ in a wind farm \\
\hline$M_{k}$ & The number of critical components considered in a wind turbine of type $k$ \\
\hline$P r_{k, n, m}$ & The failure probability of component $m$ in wind turbine $n$ of type $k$ \\
\hline$P r_{k, n}$ & The failure probability of wind turbine $n$ of type $k$ \\
\hline$L_{k, m}$ & The maintenance lead time of component $m$ in a turbine of type $k$ \\
\hline$d_{1}^{k}$ & Level 1 failure probability threshold value for turbines of type $k$ \\
\hline$d_{2}^{k}$ & Level 2 failure probability threshold value for turbines of type $k$ \\
\hline$C_{E}$ & The total expected maintenance cost per unit of time per turbine \\
\hline$\sigma_{k, n, m}$ & $\begin{array}{l}\text { Standard deviation of the predicted failure time distribution for component } m \text { in } \\
\text { wind turbine } k \text { of type } k\end{array}$ \\
\hline$\alpha_{k, m}$ & Weibull distribution scale parameter for component $m$ in a wind turbine of type $k$ \\
\hline$\beta_{k m}$ & Weibull distribution shape parameter for component $m$ in a wind turbine of type $k$ \\
\hline$T_{\operatorname{Max}}$ & The maximum simulation time \\
\hline$T_{I}$ & The inspection interval \\
\hline$c_{k, f, m}$ & The failure replacement cost for component $m$ in a wind turbine of type $k$ \\
\hline$c_{k, p, m}$ & $\begin{array}{l}\text { The variable preventive replacement cost for component } m \text { in a wind turbine of } \\
\text { type } k\end{array}$ \\
\hline$c_{k, p, T}$ & The fixed cost of maintaining a certain wind turbine of type $k$ \\
\hline$c_{\text {Farm }}$ & The fixed cost of sending a maintenance team to the wind farm \\
\hline$C_{T}$ & The total maintenance cost \\
\hline$T_{\text {LossBefore }, k, n}$ & $\begin{array}{l}\text { The number of stop days before the current inspection point for wind turbine } n \text { of } \\
\text { type } k\end{array}$ \\
\hline$T_{\text {LossAfter }, k, n}$ & $\begin{array}{l}\text { The number of stop days after the current inspection point for wind turbine } n \text { of } \\
\text { type } k\end{array}$ \\
\hline$C_{T, \text { Loss }}$ & The cost due to the loss of productivity \\
\hline$t_{A B S}$ & The current time in the simulation \\
\hline$T L_{k, n, m}$ & The real failure time for component $m$ in wind turbine $n$ of type $k$ \\
\hline$L_{\text {farm }}$ & $\begin{array}{l}\text { The maintenance lead time on the wind farm level, which is the longest lead time } \\
\text { of maintained components in the farm }\end{array}$ \\
\hline$t_{k, n, m}$ & $\begin{array}{l}\text { The current age of component } m \text { in turbine } n \text { of type } k \text { at the current inspection } \\
\text { point }\end{array}$ \\
\hline$T P_{k, n, m}$ & The predicted failure time for component $m$ in wind turbine $n$ of type $k$ \\
\hline$I F_{k, n, m}$ & $\begin{array}{l}\text { A value indicating if a failure replacement is to be performed on component } m \text { in } \\
\text { turbine } n \text { of type } k\end{array}$ \\
\hline$I P_{k, n, m}$ & $\begin{array}{l}\text { A value indicating if a preventive replacement is to be performed on component } m \\
\text { in turbine } n\end{array}$ \\
\hline$I T_{n}$ & A value indicating if a preventive replacement is to be performed on turbine $n$ \\
\hline$I F_{\text {Farm }}$ & A value indicating if a maintenance team is to be sent to the wind farm \\
\hline$t_{C I}$ & The maintenance interval in the constant-interval maintenance policy \\
\hline$C_{p, k, n, m}^{C l}$ & $\begin{array}{l}\text { The total cost of a preventive replacement for component } m \text { in turbine } n \text { of type } k \\
\text { in the constant-interval maintenance policy }\end{array}$ \\
\hline$C_{f, k, n, m}^{C I}$ & $\begin{array}{l}\text { The total cost of a failure replacement for component } m \text { in turbine } n \text { of type } k \text { in } \\
\text { the constant-interval maintenance policy }\end{array}$ \\
\hline$H m, k\left(t_{C I}\right)$ & $\begin{array}{l}\text { The expected number of failures for component } m \text { in a wind turbine of type } k \text { in } \\
\text { interval }\left(0, t_{C I}\right)\end{array}$ \\
\hline
\end{tabular}

